\title{
Optimization Models in Fire Egress Analysis for Residential Buildings
}

\author{
MICHAEL M. KOSTREVA, MALGORZATA M. WIECEK and \\ TEODROS GETACHEW
}

Department of Mathematical Sciences

Clemson University

Clemson, South Carolina 29634-1907, USA

\begin{abstract}
Fire hazard analysis often includes the use of mathematical models of the egress of the individual occupants of a structure which is involved in a fire. In this paper, we introduce some mathematical optimization models which produce as output at least one path for each occupant which is optimal with respect to some measurement. Network based models of increasing levels of complexity and realism are demonstrated by means of a sequence of examples. These examples include single attribute constant costs, single attribute timevarying costs, two attribute constant costs, and finally two attribute time-varying costs imposed on network links. Dynamic programming functional equations which are based on the principle of optimality are presented. A multi-attribute analysis is proposed to evaluate a building design with respect to evacuation paths.
\end{abstract}

KEY WORDS: Egress models, dynamic programming, networks, multiple-objective optimization.

\section{INTRODUCTION}

The mathematical modeling of egress from a building on fire falls into one of two general categories. The first is primarily descriptive, focusing on the progress of the occupants over a given time span. The second category has as its focus the determination of globally optimal trajectories of egress. Its results, useful in establishing benchmarks for actual evacuation, must draw heavily upon the theory of optimization.

Fairly extensive work has been done on the simulation of egress from a building on fire. All the models that we have looked at have included one or more of the following components. i) A network description of the building, ii) a set of heuristics for determining evacuee decisions, iii) a quantity that the evacuees are interested in minimizing, iv) input from a separate simulation that keeps track of the progress of the fire and $v$ ) an algorithm. Berlin, Dutt and Gupta [1] simulate emergency evacuation under different combinations of rescue and egress options. The required input is a set of descriptors for travel distances between various nodes in the building. As output this model gives measures of escape route congestion, the proportion of safe residents within a given time interval and the estimated evacuation time. Stahl [2] considers the effects of resident ambulatory capacities on safe egress. In EXITT, [3], [4], occupant capacities are expanded to include age, sex, and sleep; the building description has provisions for smoke detectors and background noise in addition to node and 
exit information. Occupants, having chosen a course of action, move from one node to the next according to a shortest-distance algorithm. The output is a description of the decisions and movements of occupants over time. HAZARD I, [5], [6], incorporates EXITT to simulate occupant decisions and actions, FAST [7] to perform fire and smoke calculations, and TENAB, to calculate the impact of toxins on the occupants.

Relatively little work has been done in the determination of globally optimal egress paths. EVACNET $+[8]$ is a computer program that determines time-optimal evacuation plans of a building. Taking building, occupant, node, travel time as input, it provides statistical output for quantities such as total evacuation time, number of successful evacuees, periods of building evacuation etc. It does not however trace the movement of individuals; neither does it consider people-fire interactions. The purpose of this paper is to address these two important issues.

Optimal egress involves multiple criteria decisions in a dynamic environment. Kostreva and Wiecek [9] propose that methods of multiple objective optimization and dynamic progamming may be applied to this problem. In this paper, we demonstrate applications of methods in optimal routing [10], and shortest-path algorithms in dynamic network [11] and multi-objective settings.

The body of this paper is organized into three sections. The main tool in the first section is The Principle of Optimality [12]: "An optimal policy has the property that whatever the initial state and initial decision are, the remaining decisions must constitute an optimal policy with regard to the state resulting from the first decision". First it is shown how this principle was adapted to networks, via an iterative scheme that successively approximates the optimal paths from all nodes to a destination node. It is then described how to handle the time-dependent case. Finally, this is generalized to solve the multi-objective problem, where it is of interest to optimize more than one quantity. In the Examples section, optimal egress paths in static and dynamic cost networks are obtained for a simple ranch house. Using this same building, we determine non-dominated ('best') egress paths in the two-attribute case. The subsequent section applies a multi-attribute graphical analysis to locate high and low risk zones of the ranch house.

\section{MATHEMATICAL FOUNDATIONS}

The mathematical models we consider are simplified versions of a residential building which use a superimposed network to represent locations within and around the building. The network consists of a set of nodes $\{1,2, \ldots, N\}$ and a set of links which indicate connections between nodes $\left\{\left(i_{1}, i_{2}\right),\left(i_{3}, i_{4}, \ldots\right\}\right.$. The links have a direction, indicated by the order of the indices. That is, $(3,4)$ is the link from node 3 to node 4 , while $(4,3)$ is the link from node 4 to node 3 . A path from node $i_{0}$ to node $i_{p}$ is a sequence of arcs $P=\left\{\left(i_{0}, i_{1}\right)\right.$, $\left.\left(i_{1}, i_{2}\right), \ldots,\left(i_{p-1}, i_{p}\right)\right\}$ in which the initial node of each arc is the same as the terminal arc of the preceding arc in the sequence and $i_{0}, \ldots, i_{p}$ are all distinct nodes. A path represents a connected curve along the floor of the building.

Each link carries one or more attributes (i.e. time to travel, distance to travel, etc.) which we think of as costs. The costs apply to all occupants. Each occupant in the building will be initially located at a unique node of the network, denoted an origin node. An egress path is a set of links from the origin node $i_{1}$ of the occupant to a destination node $N$, which is outside the building. That is, the egress path has the form

$$
\left\{\left(i_{1}, i_{2}\right),\left(i_{2}, i_{3}\right),\left(i_{3}, i_{4}\right), \ldots,\left(i_{r}, N\right)\right\} \text {. }
$$

The mathematical optimization models we use are designed to find, for each occupant, the egress path (not necessarily unique) which has the minimum cost, or if there are two attributes, a path which is not dominated by any other available egress path. One path $P$ is dominated by another, say $\mathrm{P}^{\prime}$, if $\mathrm{P}$ has the same or higher values in each attribute than the path $\mathrm{P}^{\prime}$. If there are several non-dominated paths for a given occupant, it is of interest to know at least one of them. 
Now a dynamic programming approach to finding optimal egress paths in a network will be described, first for the simplest case and then for the more complex models. Dynamic programming, as we will apply it, is based on the principle of optimality: A path which is optimal contains only optimal links. From an intuitive point of view, a path will be proposed initially, and this path will be improved by discarding links which are not optimal and choosing links which are optimal among the choices available.

Let the integer $\mathrm{N}$ denote the number of the destination node. We wish to compute the minimum cost path from each node $i$ to $N$. The costs to travel from node $i$ to node $j$ along link $(i, j)$ are given and are denoted $t_{i j}$. If $f_{i}$ is the optimal total cost to go from node $i$ to node $\mathrm{N}$, then the principle of optimality [10] requires that

$$
\begin{aligned}
& f_{i}=\min _{\substack{j \neq i \\
(i, j) a \text { link }}}\left(f_{j}+t_{i j}\right\} \text { for } i=1, \ldots, N-1, \\
& f_{N}=0 .
\end{aligned}
$$

One approach is to use successive approximations on the above system of nonlinear equations to compute an optimal path. This set of equations may be solved as follows:

Let $f_{i}{ }^{(0)}=\max _{j \neq i} t_{i j}$ for $i=1, . ., N-1$ and $f_{N}{ }^{(0)}=0$.

Then, for $\mathrm{k}=1,2,3, \ldots$

$$
\text { let } f_{i}{ }^{(k)}=\min \underset{\substack{j \neq i \\(i, j)}}{ }\left\{f_{j}(k-1)+t_{i j}\right\} \text { for } i=1, \ldots, N-1 \text {, }
$$

and $\mathrm{f}_{\mathrm{N}}^{(\mathrm{k})}=0$.

Now consider the case of time dependent costs $t_{\mathrm{ij}}$. A type of function which is of major interest in egress modeling is a step function. If one is coordinating an egress model with a fire physics model, such as in HAZARD I [6], the time history of smoke developing in the building is available. For example, if at time $t^{*}$, node $j^{*}$ becomes impassable due to intense smoke, then we may model this situation as the step function

$$
t_{i j *}(t)=\left\{\begin{array}{l}
t_{i j *}, \text { if } t<t^{*} \\
M, \text { if } t \geq t^{*}, \text { and } M \text { is a large positive number. }
\end{array}\right.
$$

For this type of analysis to apply one must be able to determine, for each optimal path $\left\{\left(i, i_{1}\right), \ldots,\left(j^{*}, i_{s}\right), \ldots\right\}$ the node $j^{*}$ arrival time $T_{i j}$ of the occupant with origin node $i$. There are two steps:

1) Optimize the network to compute all optimal paths from all nodes $i$ to node $N$, ignoring the step function, keeping the constant $t_{\mathrm{ij}}$. Let all paths which pass through $j^{*}$, have arrival times $\mathrm{T}_{\mathrm{ij}}$ * which are less than $\mathrm{t}^{*}$. In this case, the problem is solved and no path needs to be recomputed.

2) For each link entering the impassable node $j^{*}$ after $t^{*}$, substitute $M$ for the cost. For other links entering $j^{*}$, and for links leaving $j^{*}$, leave the costs unmodified. Optimize again. The result will be new optimal paths which avoid the impassable node, if any exist. If there are none, costs will include terms in $\mathrm{M}$.

The final type of dynamic programming we shall consider is multiple objective dynamic programming. Two or more attributes may be associated with each link of the network we are analyzing: time to travel, distance of travel, concentration of toxic chemical on the link, density of smoke, average temperature on the link, etc. In our model we seek, for each origin node, paths to the destination node which are non-dominated. In terms of the principle of optimality, non-dominated paths contain only non-dominated links. Generally, there will be a set of non-dominated paths for us to compute. Parallel to the treatment above for the single attribute case, an equation arises to describe the principle of optimality: 


$$
\left\{\underline{F}_{i}\right\}=\operatorname{vmin} \underbrace{}_{\substack{j \neq 1 \\(i, j)}}\left\{\underline{E}_{j}\right\}+t_{i j k}\} \text { for } i=1, . ., N-1,
$$

$$
\left\{\underline{\mathrm{F}}_{\mathrm{N}}\right\}=\{\underline{0}\} \text {. }
$$

In the above equation, $\left\{\mathrm{F}_{\mathrm{i}}\right\}$ stands for the set of non-dominated paths from node $i$ to node $N, t_{i j}$ is the vector of attributes associated with the link from node $i$ to node $j$ and the symbol 'vmin' stands for vector minimization, or finding the non-dominated paths. As in the earlier example, successive approximation may be used to solve these equations. Since the steps are so similar to the scalar case, they will not be repeated here. The method of dynamic programming for a similar multiple attribute network problem is discussed in [13].

The most complex model we consider is a multiple attribute network with time dependent attributes. This is the most relevant to fire egress analysis, yet it has not appeared in the literature in a useful form. Again the most relevant type of time dependence seems to be a step function, which can indicate an impassable node. The solution is by dynamic programming once again; however, it combines two of the methodologies described earlier in this section: time-dependent analysis and searching for non-dominated paths. The technical details of the extended form of dynamic programming may be found in [9]. An example to illustrate the application is contained in the next section.

\section{EXAMPLES}

The optimization models presented in the previous section are now applied to analyze the fire egress in a simple ranch house. Figure 1 depicts the floor plan of the house and nodes that have been assigned to rooms or secondary locations within rooms. It is assumed that occupants can be located at the nodes $1,2, \ldots, 6$, and can leave the house either through the door in the dining area, door in the living area or window in the bedroom. Thus, an egress path could be any path leading from node $\mathrm{i},(\mathrm{i}=1,2, \ldots, 6)$, to the destination node $\mathrm{N}=7$ located outside the house.
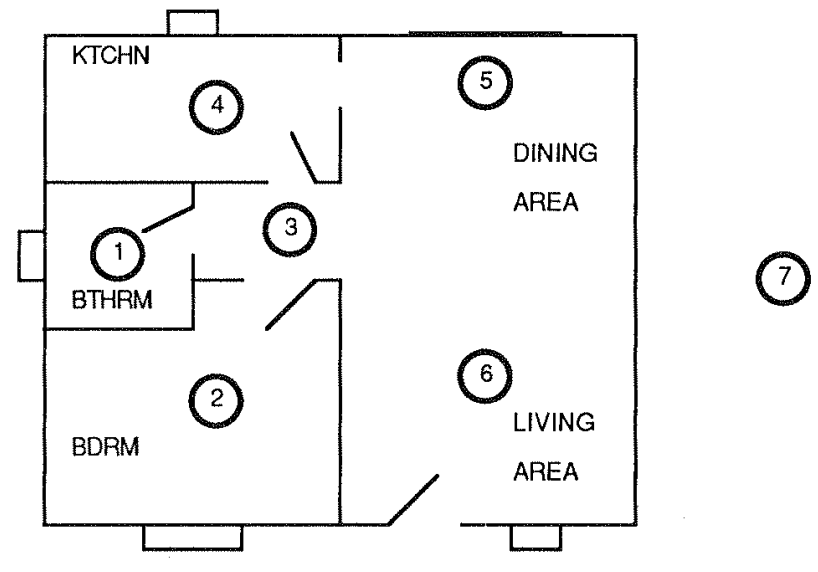

FIGURE 1. Floor plan of a ranch house with arbitrary assignment of nodes.

Figure 2 shows the egress network associated with the house. The number and direction of links results from assumed evacuation routes and directions between the rooms. 
For example, nodes 2 and 3 are connected by two links $(2,3)$ and $(3,2)$ since one can move back and forth between the bedroom and the hallway, whereas only one link connects nodes 1 and 3 since leaving the bathroom is the only movement of interest between these nodes.

Below we present four cases that illustrate the modeling concepts and show different optimal evacuation paths.

\section{Case 1: Single Attribute Network with Constant Costs.}

Figure 2 also identifies the travel cost $t_{i j}$ on each link $(i, j)$ in the network. One can interpret this cost as the (constant) amount of time necessary to travel from node $i$ to node $j$. Applying the dynamic programming approach, we can find an optimal (fastest) path from each origin node to the destination node. These optimal paths are depicted in Figure 2 and have thickened links.

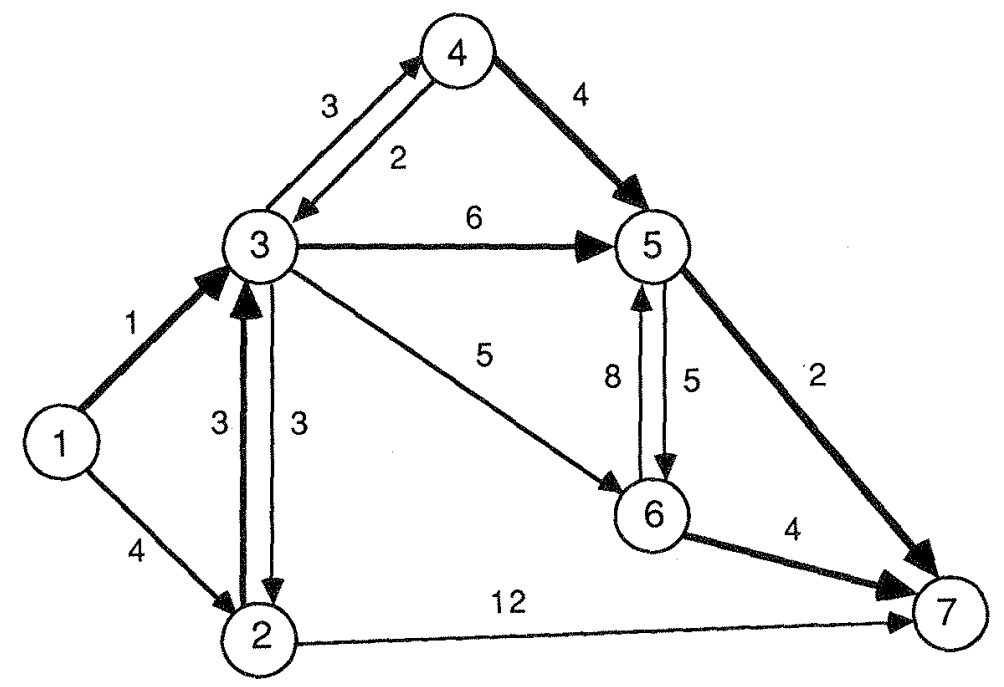

FIGURE 2. Single attribute network with constant costs and fastest paths.

The fastest paths for each origin node $\mathrm{i}=1,2, \ldots, 6$ are respectively as follows:

$\{(1,3),(3,5),(5,7)\}$,

$\{(2,3),(3,5),(5,7)\}$,

$\{(3,5),(5,7)\}$,

$\{(4,5),(5,7)\}$,

$\{(5,7)\}$,

$\{(6,7)\}$.

Consider now the network given in Figure 2 with another attribute which we can think of as (constant) distance between the nodes. Figure 4 shows the network with two attributes, time and distance, assigned to each link. Note that distances (second attribute) on each link in any pair of bilateral links are equal whereas their respective times (first attribute) do not need to be equal. Now we can find optimal (shortest) path in the network with respect to the second attribute only. They are:

$\{(1,2),(2,7)\}$, $\{(2,7)\}$, 


$$
\begin{aligned}
& \{(3,2),(2,7)\}, \\
& \{(4,5),(5,7)\}, \\
& \{(5,7)\}, \\
& \{(6,7)\} .
\end{aligned}
$$

Observe that the shortest paths are not identical with the fastest paths.

\section{Case 2: Single Attribute Network with Time Dependent Costs.}

Consider again the network given in Figure 2 and assume that node 5 is impassable. According to the analysis in the previous section one should determine the node 5 arrival time of occupant travelling from some origin node. 7,9,6,4 are the arrival times at node 5 while travelling along the fastest paths from nodes $1,2,3,4$ respectively. If node 5 becomes impassable at time $t^{*}=4$, then we solve a new fastest path problem with modified costs substituted for the costs on links $(3,5),(4,5)$ and $(6,5)$. Figure 3 depicts the original network with node 5 impassable and new optimal paths.

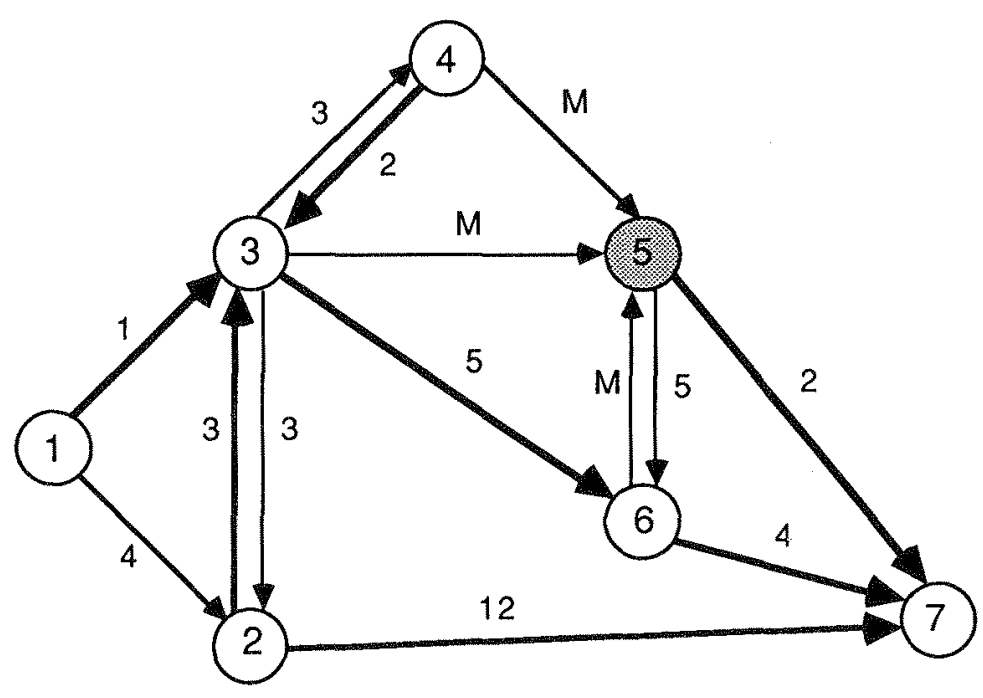

FIGURE 3. Single-attribute network with time dependent costs and new fastest paths.

The new optimal paths that avoid node 5 are:

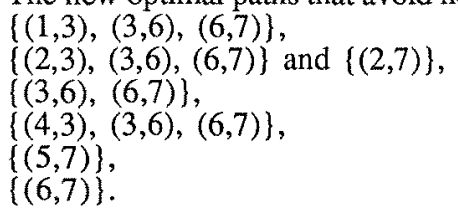

We assume that links $(5,6)$ and $(5,7)$ are available only to occupants originally located at node 5 . Hence the path $\{(5,7)\}$ is also optimal. Note that links $(4,3)$ and $(3,6)$ became optimal as a result of the new structure of the network and that there are two optimal (equally fast) paths from node 2 to node 7 avoiding node 5 . 
Case 3: Two-Attribute Network with Constant Costs.

Figure 4 illustrates also the multiple objective model discussed in the previous section if both attributes (time and distance) are associated simultaneously with each link of the network. In this case we look for non-dominated paths for each origin node that we can generate by applying vector dynamic programming. There are 11 non-dominated paths for all the origin nodes. We list them below for each origin node $\mathrm{i}$ :

$\{(1,2),(2,7)\},\{(1,3),(3,5),(5,7)\},\{(1,3),(3,4),(4,5),(5,7)\}$,

$\{(2,7)\},\{(2,3),(3,5),(5,7)\}$,

$\{(3,2),(2,7)\},\{(3,4),(4,5),(5,7)\},\{(3,5),(5,7)\}$,

$\{(4,5),(5,7)\}$,

$\{(5,7)\}$,

$\{(6,7)\}$.

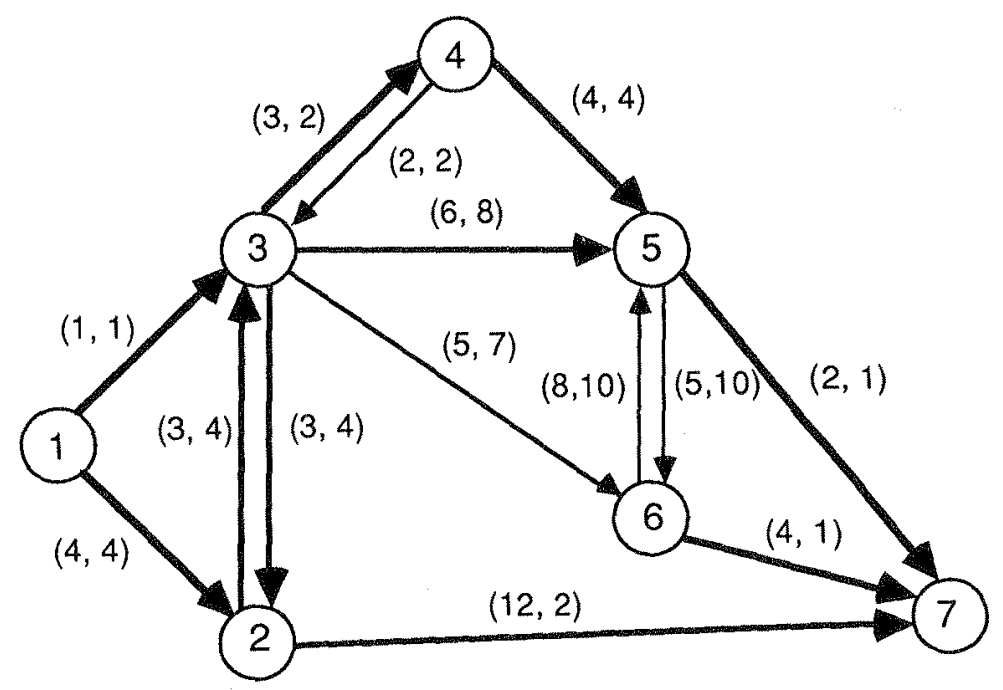

FIGURE 4. Two-attribute network with constant costs and non-dominated paths.

Notice that for nodes 1,2 , and 3 additional paths have been discovered by the multiattribute network model. These were not found in the single attribute model solution.

Case 4: Two-Attribute Network with Time Dependent Costs.

The final case considered includes a two-attribute network and a step function imposed on node 5 with $t^{*}=4$. Figure 5 depicts the network with impassable node 5 and adjusted costs on links leading to this node. follows:

The vector dynamic programming approach generates 9 non-dominated paths as

$\{(1,2),(2,7)\},\{(1,3),(3,6),(6,7)\}$,

$\{(2,7)\}$,

$\{(3,2),(2,7)\},\{(3,6),(6,7)\}$,

$\{(4,3),(3,2),(2,7)\},\{(4,3),(3,6),(6,7)\}$, 
$\{(5,7)\}$ only for occupants originally located at node 5 , $\{(6,7)\}$.

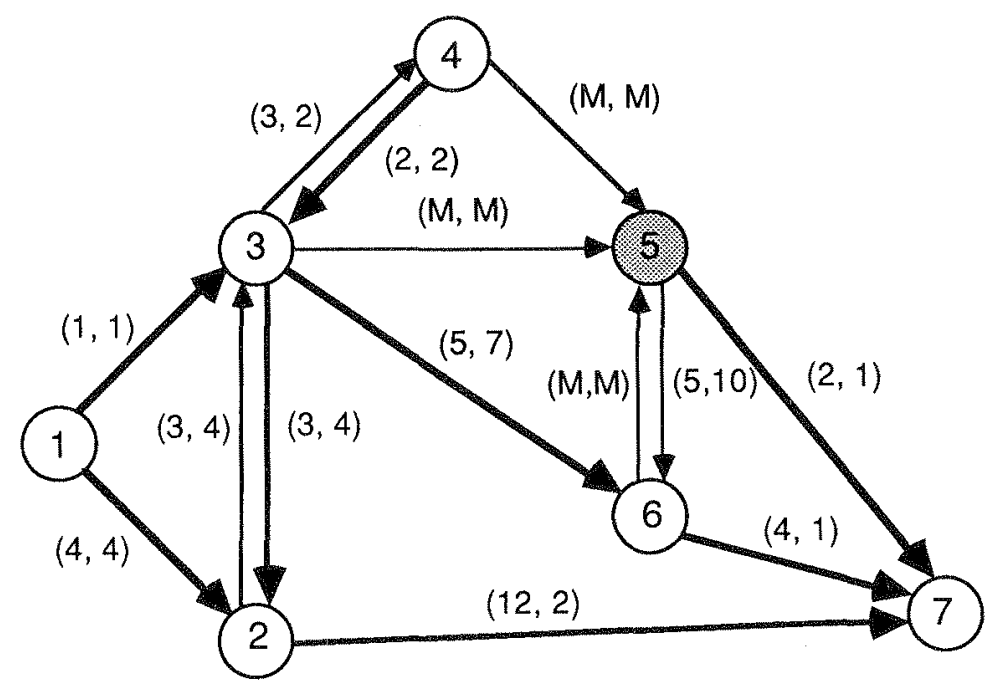

FIGURE 5. Two-attribute network with time dependent costs and non-dominated paths.

Note two new non-dominated links $(4,3)$ and $(3,6)$ that contribute to the new nondominated paths in the network. The presence of the very costly links to the impassable node 5 has caused a revision to the set of solution paths.

\section{DISCUSSION AND ANALYSIS}

Solving the optimal path problems in the preceeding section resulted in different solutions that depend on the concept of optimality assumed for each optimization model.

The models were developed for one network whose nodes and links identify all potential egress routes in the house. Number and location of nodes and links are, of course, arbitrary but should agree with physical locations of occupants and their movement, location of decision points (e.g. doorways) and final exit. Developing a network for the house is the first major phase of the modeling process.

The multi-attribute model allows for simultaneous analysis of several costs that illustrate the multiple objective nature of the evacuation process. Moreover, given nondominated paths by applying a multi-attribute analysis one can obtain a fresh insight into a building design with respect to the location of a fire.

In order to perform the analysis one can plot non-dominated paths in the two-attribute space. Figure 6 depicts the all non-dominated paths found in the two-attribute network with constant costs and time dependent costs. The origin node number assigned to each nondominated path helps identify the low and high risk zones within the house. The paths originating at node 2 (of cost $(11,13)$ ) and node 1 (of cost $(16,6)$ ) belong to the high risk zone due to the large values of time and distance to travel. The paths from node 5 and 6 are, of course, in the lowest risk zone. When node 5 becomes impassable, the path from node 6 stays in the lowest risk zone, but the only two paths leaving node 4 (of cost $(11,10)$ and 
$(17,8))$ are in the high risk zone. Thus, one may conclude that a fire in the dining area of the house makes the evacuation from the kitchen very difficult.

Assuming that one node at a time becomes impassable, we can construct multiattribute graphs, as in Figure 6, for each node of the network. We can then determine the high and low risk zones in the house for any location of the fire. Such a classification could have a new impact on the design of residential houses in terms of structure, arrangement, and location of rooms and hallways. Furthermore, occupants' decisions and actions to be taken during the evacuation could be motivated by the study of the high and low risk zones in the house.

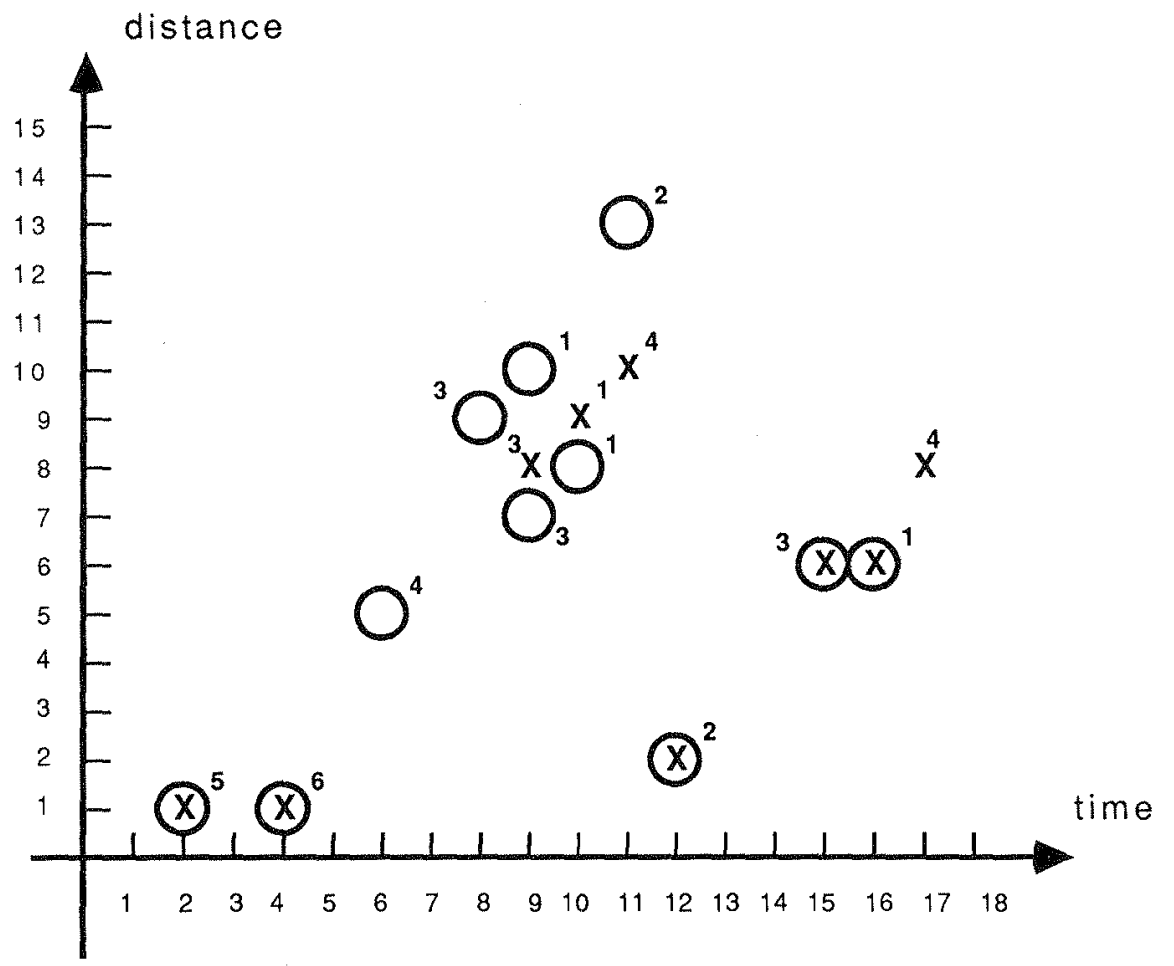

FIGURE 6. Costs of non-dominated paths for two-attribute network with constant

costs ( $O$ ) and time dependent costs $(\mathbf{X})$ with origin node numbers.

\section{CONCLUSIONS}

A mathematical optimization approach provided by dynamic programming allows us to simultaneously find optimal egress paths for all occupants in a residential building. Time varying network attributes, as well as multiple attributes on each link of a network, may be handled in the same framework. Decision making in a dynamic environment which includes conflicting goals more faithfully reflects the situation faced by the occupants of a residential building which is involved in a fire.

From the optimal solutions we may gain several insights not available in earlier 
analyses. Revised paths, which may be quite instructive, are obtained. The amount of time or distance by which the optimal paths increase when a room becomes impassable may be computed. All non-dominated paths for the building may be plotted in multi-attribute graphs which allow for classification and a new way to view a building.

The optimization models developed in this paper are of special interest for the Center for Fire Research (CFR) at the National Institute of Standards and Technology. The dynamic programming approach relates very well to EXITT [3], [4], and HAZARD I [5], [6], developed at CFR and so there is a possibility of incorporating this methodology in the simulation models of CFR.

\section{ACKNOWLEDGEMENT}

This research was supported by Grant No 60NANB0D1023 from the Center for Fire Research, National Institute of Standards and Technology, Gaithersburg, Maryland, U.S.A.

\section{REFERENCES}

1. Berlin, G. N., Dutt, A. and Gupta, S. M., "Modeling Emergency Evacuation from Group Homes," Fire Technology, 18, 38-48, 1982.

2. Stahl, F. I., "BFIRES-II: A Behavior Based Computer Simulation of Emergency Egress During Fires," Fire Technology , 18, 49-65, 1982.

3. Levin, B. M., "EXITT - A Simulation Model of Occupant Decisions and Actions in Residential Fires: User's Guide and Program Description," NBSIR 87-3591, Center for Fire Research, National Bureau of Standards, Gaithersburg, Maryland, 1987.

4. Levin, B. M., "EXITT - A Simulation Model of Occupant Decisions and Actions in Residential Fires," in Fire Safety Science -Proceedings of the Second International Symposium. International Association of Fire Safety Science. Tokyo, June 13-17. 1988, Hemisphere Publishing Company, New York, 1989.

5. Bukowski, R. B., Peacock, R. D., Jones, W. W. and Forney, C. L.,"Technical Reference Guide for HAZARD I, Fire Hazard Assessment Method," Handbook 146, Volume II, U. S. National Institute of Standards and Technology, Gaithersburg, Maryland, 1989.

6. Peacock, R. D. and Bukowski, R. W., "A Prototype Methodology for Fire Hazard Analysis," Fire Technology, 26, 15-40, 1990.

7. Jones, W. W. and Peacock, R. D., "Refinement and Experimental Verification of a Model for Fire Growth and Smoke Transport", in Fire Safety Science - Proceedings of the Second International Symposium, International Association of Fire Safety Science. Tokyo, June 13-17, 1988, Hemisphere Publishing Company, New York, 1989.

8. Kisko, T. M. and Francis, R. L., "EVACNET+: A Computer Program to Determine Optimal Building Evacuation Plans," Fire Safety Journal , 9, 220, 1985.

9. Kostreva, M. M. and Wiecek, M. M., "Time Dependency in Multiple Objective Dynamic Programming," Department of Mathematical Sciences, Clemson University Technical Report \#601, Clemson, South Carolina, 1991.

10. Bellman, R., "On a Routing Problem," Quarterly of Applied Mathematics, 16, 87-90, 1958.

11. Cooke, K. L. and Halsey, E., "The Shortest Route Through a Network with TimeDependent Internodal Transit Times," Journal of Mathematical Analysis and Applications. 14, 493-498, 1966.

12. Bellman, R, and Kalaba, R., Dynamic Programming and Modern Control Theory, Academic Press, New York, 1965.

13. Daellenbach, H. G. and De Kluyver, C. A., "Note on Multiple Objective Dynamic Programming," Journal of the Operational Research Society, 31, 591-594, 1980. 